\title{
BreastCare
}

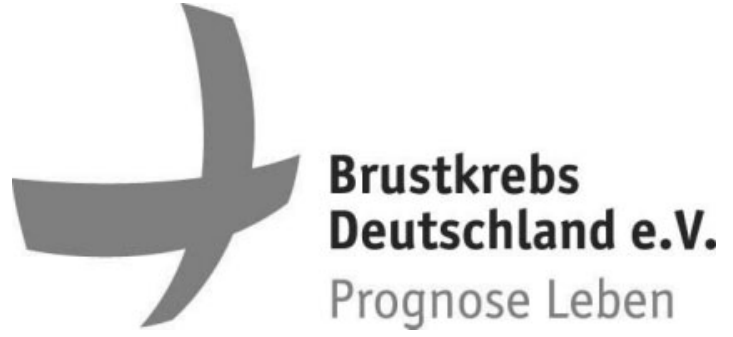

Brustkrebs Deutschland e.V.

Charles-de-Gaulle-Str. 6, 81737 München

Tel. 0894161 98-00, Fax -01

E-Mailinfo@dbkh.de

www.brustkrebsdeutschland.de

\section{Brustkrebs Deutschland e.V.}

Im Verein Brustkrebs Deutschland e.V. haben Brustkrebsbetroffene, Angehörige, Ärzte und auch nicht Betroffene gemeinsame Ziele: die Öffentlichkeit aufrütteln und für dieses wichtige Thema sensibilisieren, die Bedeutung der Früherkennung immer wieder zu betonen, viele Informationen bereit $\mathrm{zu}$ stellen, und betroffene Frauen und deren Familien zu unterstützen.

Der Verein bietet eine breite Basis, auf der sich alle Beteiligten austauschen und neue Wege und Ziele erarbeiten können. Es wird sowohl die Kommunikation zwischen allen Beteiligten als auch die Weitergabe von verständlichen Informationen im Bereich Brustkrebs gefördert. Der Verein hat sich zum Ziel gesetzt, durch Aufklärung und Information Frauen aller Alterstufen aufzufordern, für ihren eigenen Körper und Ihre Gesundheit die Verantwortung zu übernehmen. Indem Sie sich frühzeitig über die Krankheit informieren, zu den angebotenen Untersuchungen gehen und ihren Körper wahrnehmen, ermöglichen sie die Früherkennung der Krankheit und verbessern damit die Heilungschancen.

Die Website: www.brustkrebsdeutschland.de bietet ein innovatives interaktives Programm mit laienverständlichen Informationen rund um das Thema Brust und Brustkrebs. Dieses Programm wird zurzeit erweitert und mit neuen Inhalten ergänzt. Kongressberichte, verfasst von Brustkrebsspezialisten, mit den Neuigkeiten aller wichtigen deutschen und internationalen Kongresse (z.B. Deutscher Krebskongress, Kongress der Deutschen Gesellschaft für Senologie, European Breast Cancer Conference (EBCC) American Society of Clinical Oncology (ASCO), European Society of Medical Oncology (ESMO) und European Cancer Conference (ECCO) und die San Antonio Breast Cancer Conference (SABCS) erweitern mit aktuellen Informationen dieses Angebot. Interviews mit führenden Spezialisten zu den verschiedenen Themen rund um Brustkrebs bieten einen tieferen Einblick in sehr komplizierte Teilgebiete.

Eine Telefonhotline bietet die Möglichkeit, mit anderen betroffenen Frauen zu sprechen und sich weitere Informationen zu holen: 089416198 00. Informationsveranstaltungen rund um das Thema ermöglichen es betroffenen Frauen, Angehörigen und Interessierten sich auf den neuesten Stand der Wissenschaft zu bringen, sich untereinander auszutauschen und mit führenden Spezialisten zu diskutieren. Öffentlichkeitswirksame Aktionen sollen das Bewusstsein für Brustkrebs fördern und auch die politische Arbeit des Vereins unterstützen.

Wir fordern ein flächendeckendes Krebsregister in Deutschland, damit die Qualität der Behandlung und Fortschritte in der Medizin überprüft werden können. Das qualitätsgesicherte Mammografiescreening gibt es leider immer noch nicht in ganz Deutschland und wir fordern, dass nur noch erfahrene Spezialisten die systemische und somit komplizierte Erkrankung Brustkrebs operieren und nach dem neuesten Stand der Wissenschaft behandeln dürfen. Hierzu gehört auch eine gute interdisziplinäre Anbindung und Zusammenarbeit.

Der Verein wird von einem hervorragend besetzten Fachbeirat unterstützt, sowie von prominenten Botschaftern, wie den Schauspielerinnen Andrea L'Arronge und Christiane Krüger, der Chefredakteurin von «Bunte», «InStyle» und «Amica» Patricia Riekel, den Autorinnen und Journalistinnen Andrea Sixt und Janine White sowie dem Schauspielerehepaar Carolin Fink und Michael Mendl. 